Article

\title{
Observation of Sintering of Clathrate Hydrates
}

\section{Tsutomu Uchida *, Toshiki Shiga, Masafumi Nagayama and Kazutoshi Gohara}

Division of Applied Physics, Faculty of Engineering, Hokkaido University, N13 W8, Kita-ku, Sapporo 060-8628, Japan; E-Mails: koujoushin.haitei.kokushimusou @ docomo.ne.jp (T.S.); macci@eng.hokudai.ac.jp (M.N.); gohara@eng.hokudai.ac.jp (K.G.)

* Author to whom correspondence should be addressed; E-Mail: t-uchida @eng.hokudai.ac.jp; Tel.: +81-11-706-6635; Fax: +81-11-706-6635.

Received: 25 October 2010; in revised form: 2 December 2010 / Accepted: 10 December 2010 / Published: 13 December 2010

\begin{abstract}
Clathrate hydrates have recently received attention as novel storage and transportation materials for natural gases or hydrogen. These hydrates are treated as powders or particles, and moderate storage temperatures (around $253 \mathrm{~K}$ ) are set for economic reasons. Thus, it is necessary to consider the sintering of hydrate particles for their easy handling because the hydrates have a framework similar to that of ice, even though their sintering would require guest molecules in addition to water molecules. We observed the sintering process of clathrate hydrates to estimate the rate of sintering. Spherical tetrahydrofuran (THF) hydrate particles were used in observations of sintering under a microscope equipped with a CCD camera and a time-lapse video recorder. We found that THF hydrate particles stored at temperatures below the equilibrium condition sintered like ice particles. The sintering part was confirmed to be not ice, but THF hydrate, by increasing the temperature above $273 \mathrm{~K}$ after each experiment. The sintering rate was lower than that of ice particles under the normal vapor condition at the same temperature. However, it became of the same order when the atmosphere of the sample was saturated with THF vapor. This indicates that the sintering rate of THF hydrate was controlled by the transportation of guest molecules through the vapor phase accompanied with water molecules.
\end{abstract}

Keywords: sintering; tetrahydrofuran hydrate; ice; vapor transportation 


\section{Introduction}

Natural gas hydrates (NGHs) located under the deep sea or permafrost are a potential energy source. For example, deposits of NGHs have been reported in marine sediments in the Nankai Trough off the Pacific coast of Japan, where the water depth is more than $500 \mathrm{~m}$ [1]. Estimates suggest that the reserves of NGHs correspond to several decades of supply of natural gas to Japan [2], making it an important potential source of energy. Currently, natural gas is transported from a production site to the place of consumption mainly by a liquefied natural gas (LNG) system. However, for economic reasons an LNG system can only be applied to large gas fields. The increasing demand for natural gas requires the development of systems to transport natural gas that are more economical and practical and can be applied to small and/or medium size gas fields. An NGH system is a promising solution because hydrates can contain a large amount of gases under thermal conditions milder than those of an LNG system. Recently, a continuous NGH system has been developed to efficiently store and transport natural gases using hydrate pellets $[3,4]$.

In addition to the natural gas carrier, attention has also been paid to clathrate hydrates as a novel carrier of hydrogen $\left(\mathrm{H}_{2}\right) . \mathrm{H}_{2}$ hydrate has a type-II structure in the pressure range of 0.18 to $0.22 \mathrm{GPa}$ at a temperature of $249 \mathrm{~K}$ [5]. It was recently found that the addition of a second guest substance, such as tetrahydrofuran (THF) [6-10], quaternary ammonium salts [8,11,12], cyclohexanone [13] or amines [14] is effective in lowering the stability pressure. However, this technique is still highly discussed because the addition of second guests reduces the overall $\mathrm{H}_{2}$ storage capacity. Most researchers have reported that the maximum amount of $\mathrm{H}_{2}$ stored in $\mathrm{THF}+\mathrm{H}_{2}$ hydrates is at most $1 \mathrm{wt} \%[10,13,15-17]$, which is a fourth of that in a pure $\mathrm{H}_{2}$ hydrate. If the $\mathrm{H}_{2}$ storage capacity is improved up to that expected in pure $\mathrm{H}_{2}$ hydrate, the utilization of clathrate hydrates becomes realistic as a future engineering technology [18]. In fact, the technique has been developed to increase the $\mathrm{H}_{2}$ storage to $3.4 \mathrm{wt} \%$ in $\mathrm{THF}+\mathrm{H}_{2}$ hydrate [19].

Since the materials are treated as powder or pelletized particles and the storage conditions are set at around $253 \mathrm{~K}$, moderate temperatures below the melting point of ice, and atmospheric pressure for economic reasons, it is necessary to consider the sintering of hydrate particles for their easy handling. Sintering is a thermal treatment for bonding particles to a coherent, predominantly solid structure via mass transport events that often occur on the atomic scale. The bonding leads to improved strength and lower system energy [20,21]. Sintering is observed for snow and ice particles, especially when they are stored at temperatures near the melting point. Clathrate hydrates have a framework similar to that of ice, and thus, sintering may occur even though not only water molecules but also guest molecules are required. However, experimental investigations of sintering on clathrate hydrates are very limited [22]. In the present study, we observed the sintering processes of clathrate hydrates to estimate how fast it progressed under several conditions below the ice point.

\section{Results and Discussion}

Optical microscopic images of ice particles (approximately $2 \mathrm{~mm}$ in diameter) showed sintering during storage at $263.1 \mathrm{~K}$ for $6 \mathrm{~h}$ (Figure 1). The necks of the particles grew from the contact points, while the surfaces of particles became smooth. 
The microscopic observations of THF hydrate particles indicated the sintering occurred at similar conditions for ice particles (263.2 K and 0.1 MPa, Figure 2); however, the growth rate of the necks was lower than that for ice. We confirmed that the sintering part was not ice but THF hydrate by increasing the temperature above $273.2 \mathrm{~K}$ after the completion of each experiment.

Figure 1. Sintering of ice particles at $263.1 \mathrm{~K}$ : (a) initial condition and (b) $6 \mathrm{~h}$ after contact. Scale bar indicates $1 \mathrm{~mm}$. Red allow heads indicate the edges of sintering neck.
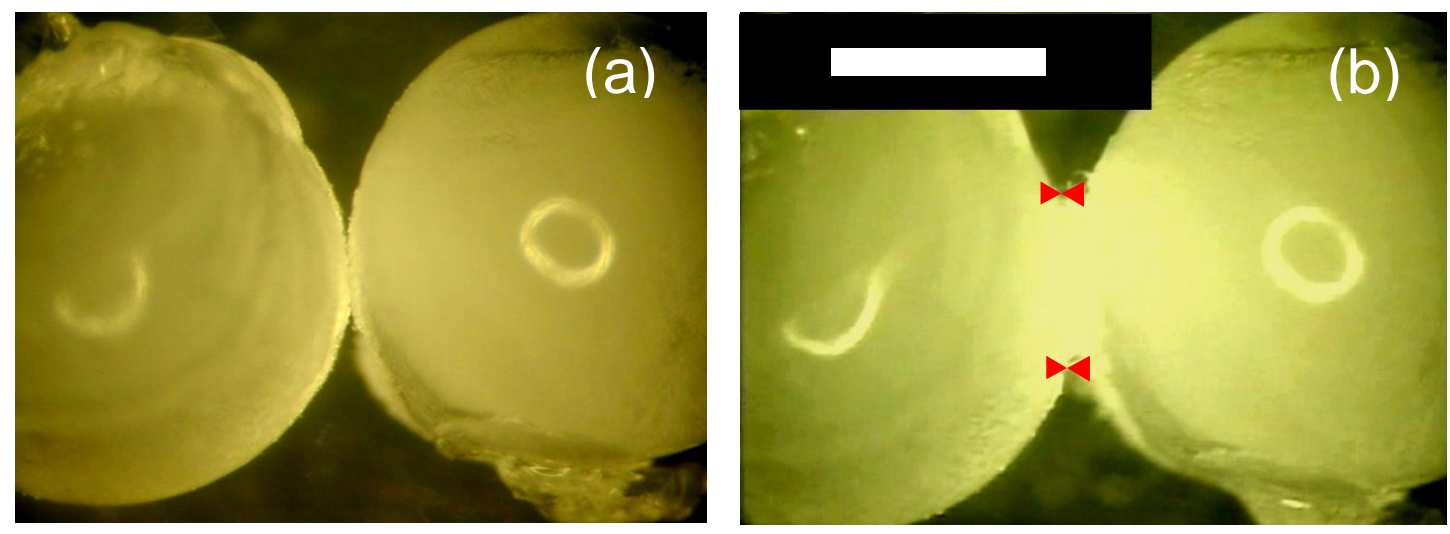

Figure 2. Sintering of THF hydrate particles at $263.2 \mathrm{~K}$ : (a) $10 \mathrm{~min}$ after contact and (b) $6 \mathrm{~h}$ after contact. Scale bar indicates $1 \mathrm{~mm}$. Red allow heads indicate the edges of sintering neck.
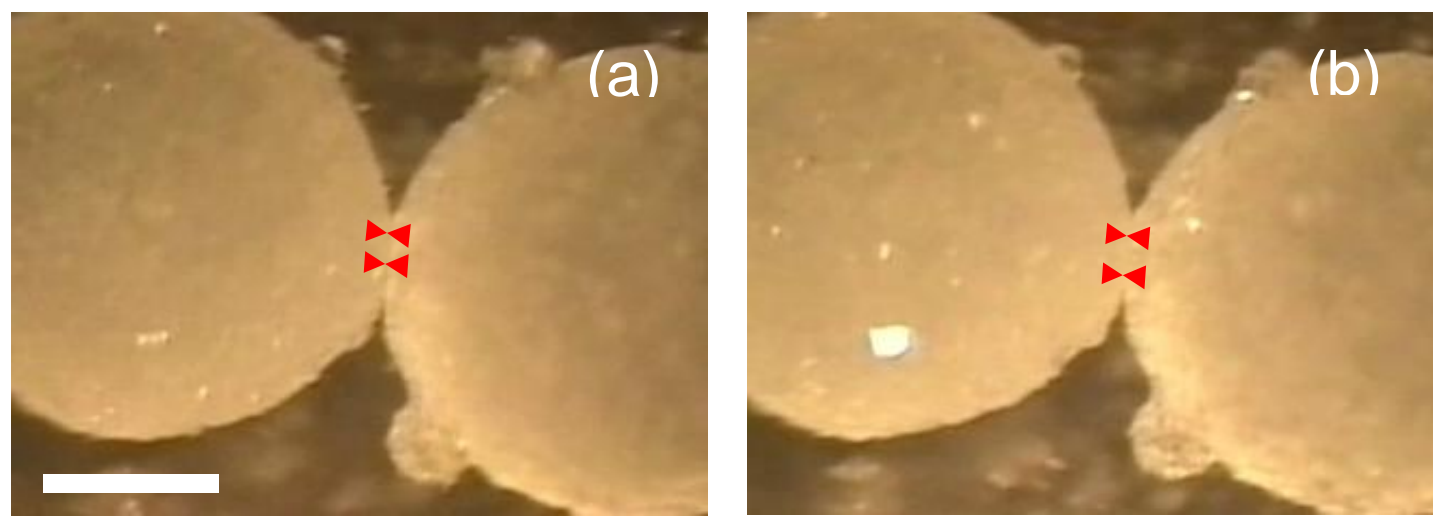

To compare the sintering rates quantitatively, we measured the size ratio between the neck radius $x$ and the particle radius $r$ as a function of time. The sintering rate is usually expressed as:

$$
(x / r)^{q}=\mathrm{Cr} r^{-p} t
$$

where $t$ is time, $\mathrm{C}$ is a parameter which captures material and geometric constants, and $p$ and $q$ are numerical constants depending on the working mechanism [21,23]. The logarithmic relationship of $x / r$ with $t$ for the results obtained in the present study (Figure 3 ) indicates that both the sintering of ice and THF hydrate particles follow Equation (1). Figure 3a shows that the sintering rates of ice particles coincided well with the rates obtained in previous studies [24,25]. These agreements validate our experimental procedures. Previous studies [23] and the present observations indicate that the main rate-determining process of ice-particle sintering under the present experimental conditions was $\mathrm{H}_{2} \mathrm{O}$ molecule transportation through the vapor phase. 
Figure 3. Relative neck growth $x / r$ ( $x$ : neck radius, $r$ : average particle radius) versus time $t$ for (a) ice particles and (b) THF hydrate particles. The data from [25] is drawn as the average of three experiments at $263.2 \mathrm{~K}$.

(a)

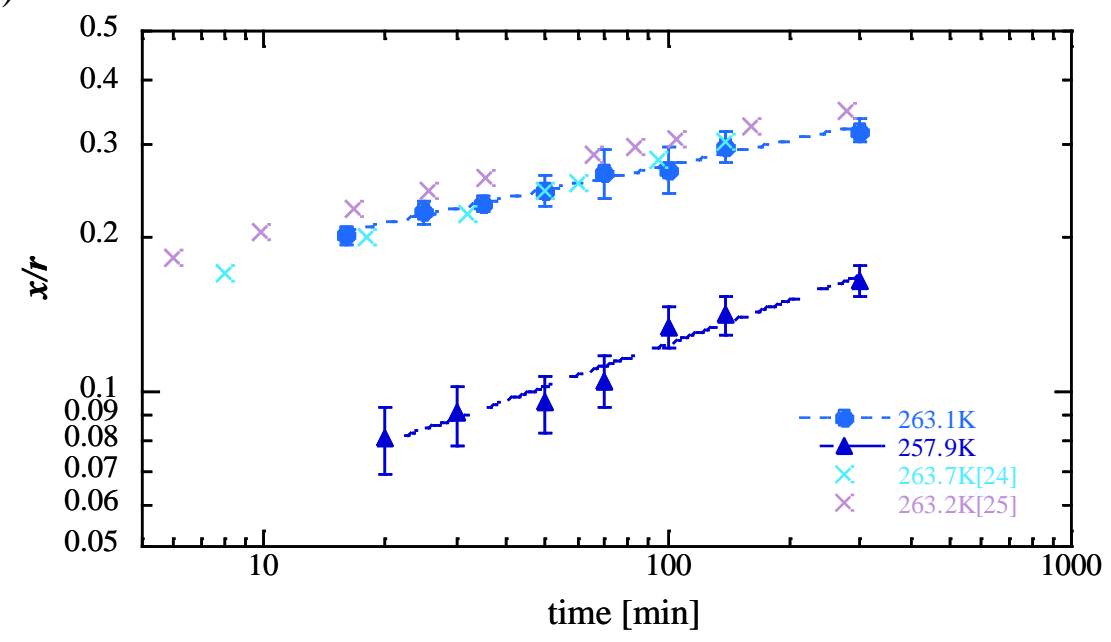

(b)

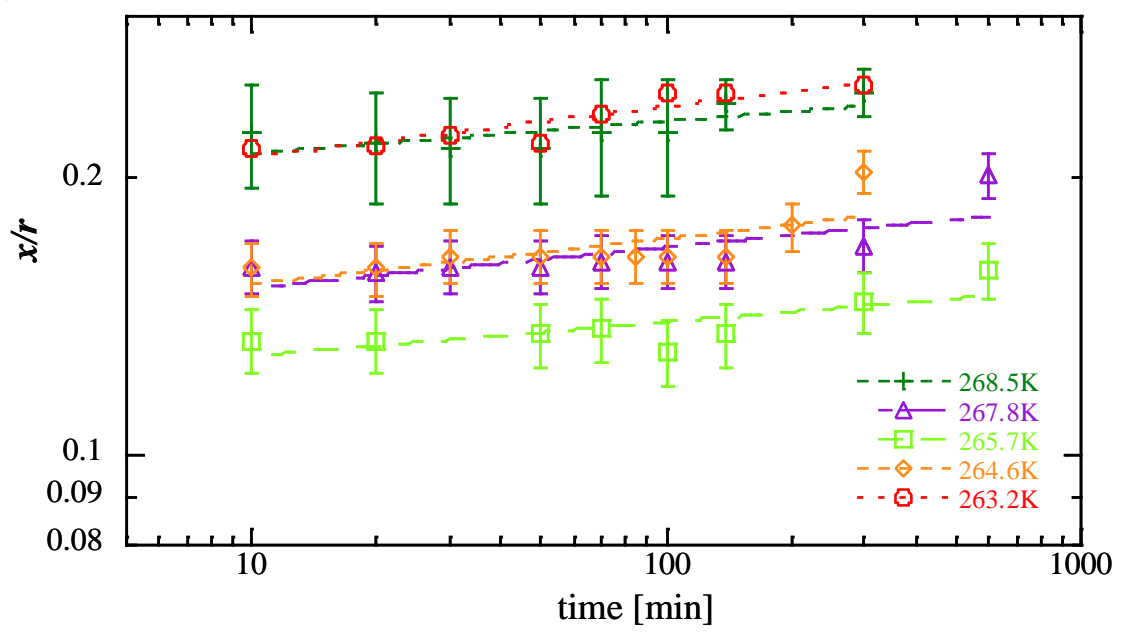

The sintering rates for THF hydrates were less than those for ice particles (Figure $3 b$ ). The time constant $q^{-1}$ was approximately 0.055 for THF hydrates and 0.16 for ice particles at $263 \mathrm{~K}$ (see Table 1). Although the surfaces of THF hydrate particles were smoothed during sintering (it can be seen that the reflection of the light increased in Figure $2 b$ ), the values of $q^{-1}$ for THF hydrates were much smaller than expected for the vapor transportation mechanism [21]. If we assume that the rate-determining process for THF hydrate particles is the vapor transportation mechanism under the present experimental conditions, we consider that the lower sintering rate for THF hydrates than for ice would result from a lack of guest molecules being supplied for the clathrate hydrate formation at the neck because it requires both guest and host molecules.

To investigate the sintering process mentioned above, we carried out similar experiments under almost saturation conditions with THF vapor in the reaction cell. The THF hydrate particles were set in the sample cell, which was in turn immersed in THF solution. The microscopic images of THF particles are shown in Figure 4 and their sintering rates in Figure 5. The figures show the sintering rates 
that were obviously higher than those of previous experiments under the normal vapor conditions (Figures 2 and $3 b$ ) at the same temperatures.

Figure 4. Sintering of THF hydrate particles under saturation condition in vapor at $262.2 \mathrm{~K}$ : (a) $10 \mathrm{~min}$ after contact and (b) $6 \mathrm{~h}$ after contact. Scale bar indicates $1 \mathrm{~mm}$. Red allow heads indicate the edges of sintering neck.
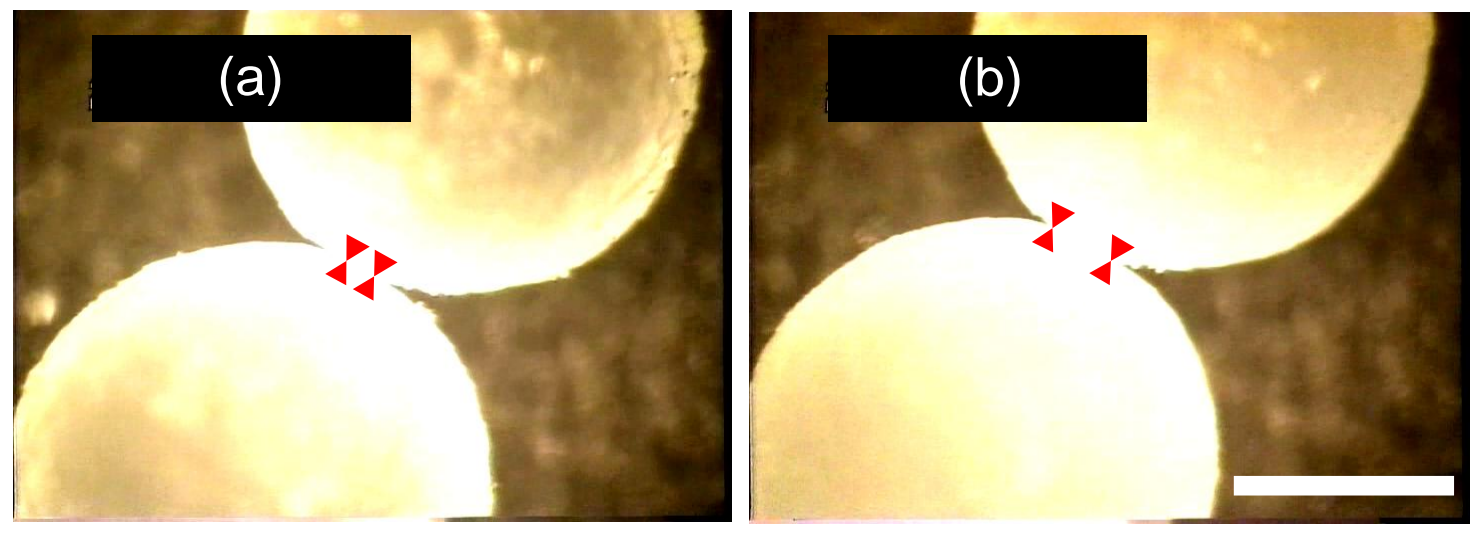

Figure 5. Relative neck growth $x / r$ ( $x$ : neck radius, $r$ : average particle radius) versus time $t$ for THF hydrate particles when the atmosphere was saturated with THF vapor. For comparison, experiments for ice at $263.1 \mathrm{~K}$ (right blue solid circles) and for THF hydrate under the normal vapor condition at $263.2 \mathrm{~K}$ (red open circles) are also plotted.

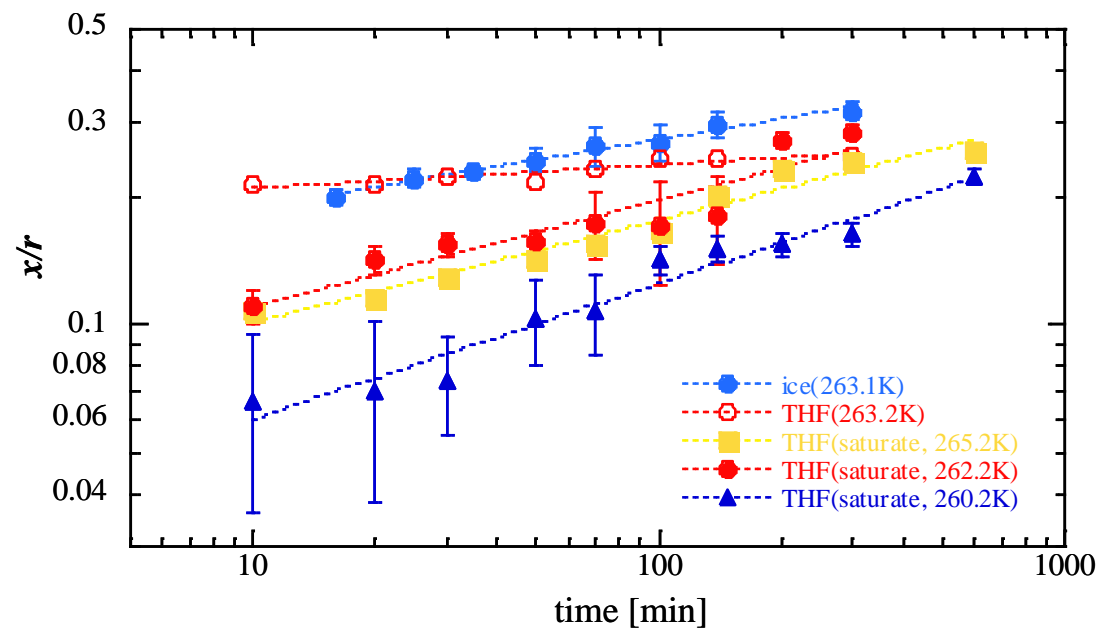

The time constant of the sintering rate of THF hydrates under saturation conditions was approximately 0.25 (Figure 6), within the temperature range of the present study. This time constant was approximately five times that for THF hydrates under the normal vapor condition and almost of the same order as that for ice particles at similar temperature. Blackfold [21] summarized previous works discussing about the relation between the value of the time constant and the dominant mass transport mechanisms. Although the estimation of the time constant related to each suggested mechanism has not been agreed upon yet, the estimated value for both ice and THF hydrate under saturated conditions are belong to the range of the time constants suggested previously [21] (shown by hatched area in Figure 6). Therefore, it is considered that the sintering rate of THF hydrates was mainly 
controlled by the supplementing of guest molecules from the vapor phase accompanied with the host water molecule transportation.

Figure 6. Temperature dependence of $q^{-1}$ for ice (blue solid circle) and THF hydrates (normal vapor condition: red open circle, THF saturated vapor conditions: red solid circle). The hatched area indicates the expected $q^{-1}$ value for the vapor transportation mechanism [21].

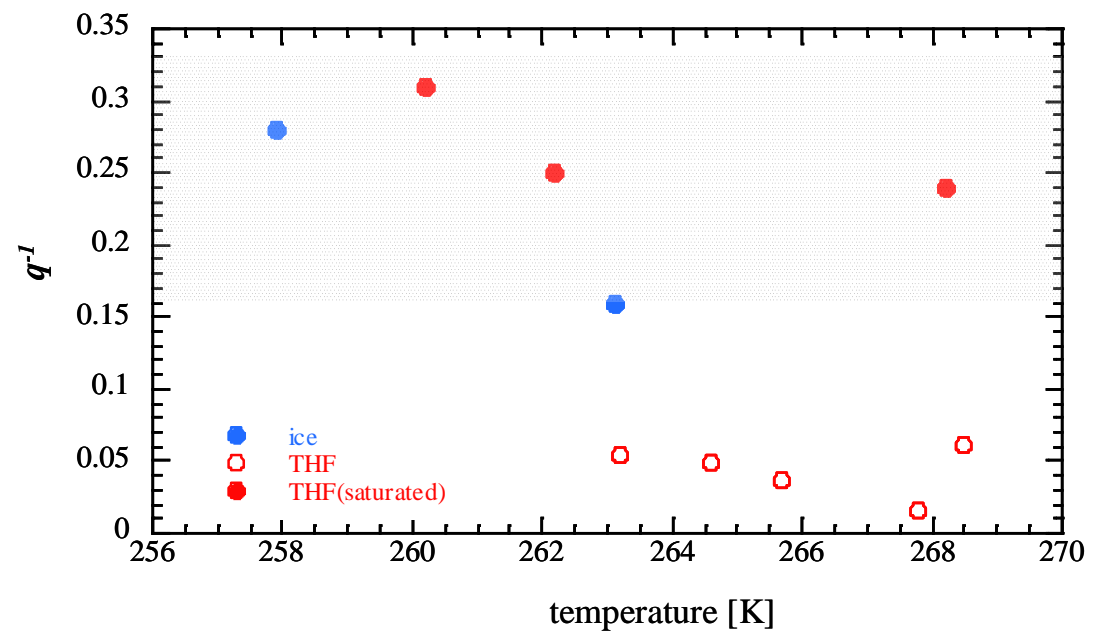

Thus the supposed process of THF hydrate sintering is illustrated in Figure 7. The early stage of sintering is the molecular transportation to the contact point mainly by surface diffusion [23]. However, we could not observe this process owing to low spatial resolution of the experimental setup. In the following process under the storage condition, the THF hydrate sublimated when the vapor was undersaturated with $\mathrm{H}_{2} \mathrm{O}$ and THF.

Figure 7. Schematic illustration of sintering of THF hydrate particles.

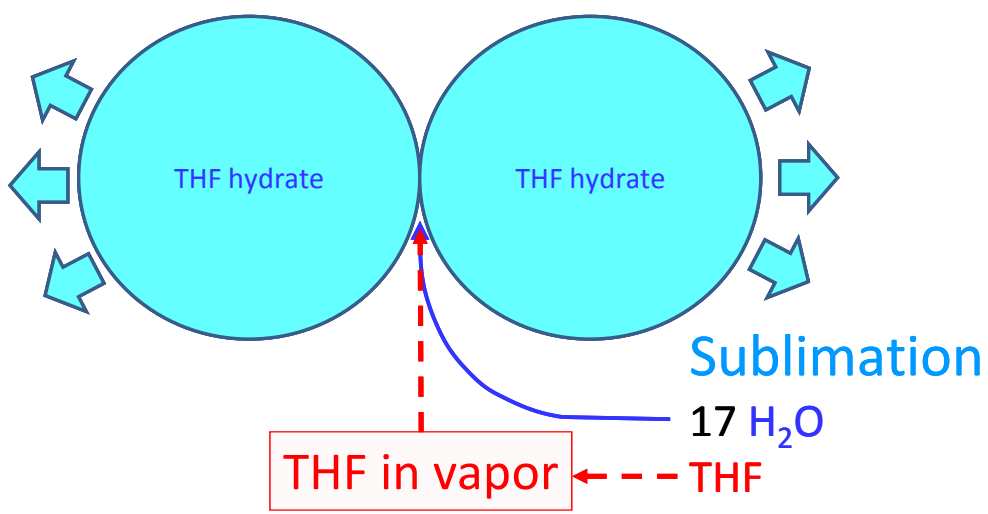

The sublimation was observed under a microscope by the gradual increase in reflection from the surface of particles. Since the molecular composition of the original THF solution was THF: $\mathrm{H}_{2} \mathrm{O}=1: 17$, and the formed THF hydrates were usually polycrystals with some composition variations heterogeneously, the molecules emitted into the surrounding atmosphere are considered to be $\mathrm{H}_{2} \mathrm{O}$ rich (the THF concentration would be less than 5.5 mol\%). The $\mathrm{H}_{2} \mathrm{O}$ molecules moved to the sintering site under the concentration gradient according to the Gibbs-Thomson effect. If THF 
molecules were saturated in the vapor phase under the storage condition, they would also be transferred easily to the sintering site to form THF hydrate. This would be the case in the sintering experiment of THF hydrates in saturated THF vapor. Under this condition, THF hydrate sintered as quickly as ice. However, the experiments without THF solution around the particles had much lower sintering rates. This is explained by the lack of THF molecules to form THF hydrate at the sintering site. Although we do not have enough data of the saturated vapor pressure of THF at temperatures below $273.2 \mathrm{~K}$ in the presence of THF hydrate, the results obtained in the present study indicate that THF molecules emitted only by sublimation were insufficient to grow the sintering neck.

On the basis of this THF hydrate sintering process, we can consider the storage and transportation processes of NGHs. Although the sintering rate is lower than that of ice, gas hydrates also undergo sintering when they are stored for a significant period at temperatures below the melting point of ice. Since the sintering of gas hydrate particles requires the supplement of both host $\mathrm{H}_{2} \mathrm{O}$ and guest gas molecules, the sintering of gas hydrates was controlled by the vapor concentration of both molecules. In other words, the rate of sintering depends on the vapor pressure of guest or host molecules. However, if the hydrate particles are coated by ice to emphasize the self-preservation effect, such as in the case of hydrate pellets [25], the sintering process would be similar to that of ice because sintering occurs mainly at the surface of the sample.

\section{Experimental Section}

We prepared a spherical THF hydrate to observe sintering under a microscope. A droplet of the THF solution prepared from 99.5\% THF (Wako Pure Chemical Industries, Ltd.) and de-ionized distilled water with a stoichiometric concentration (THF: $\mathrm{H}_{2} \mathrm{O}=1: 17$ in mole fraction) was rapidly frozen in liquid nitrogen. The quench process made the sample a polycrystal containing very fine grains with random crystal orientation. Therefore, we eliminated the effect of crystal orientation on the sintering rate reported by Hobbs and Mason [26]. Then the particles annealed at 274-275 K to make a small spherical particle of THF hydrate $(2-3 \mathrm{~mm}$ in diameter). At the same time, the very fine ice grains would be consumed for the recrystallization of THF hydrate polycrystal. THF hydrate particles were stored in a temperature-controlled cell placed on the stage of a microscope (Nikon FMZ-10) equipped with a CCD camera (Olympus CS230B) and a time-lapse S-VHS video recorder (Victor SR-S990). The lighting of the sample was Nikon cold-fiber optics light system to eliminate the temperature increase during the experiment. Two particles were selected to observe the sintering in the experiment, and remaining other particles worked for maintaining a stable atmosphere in the cell. A schematic diagram of the experimental setup is shown in Figure 8. The temperature of the sample cell was controlled at above $260 \mathrm{~K}$ within $\pm 0.2 \mathrm{~K}$.

The video images were analyzed using an image analyzing software (Fuji Photo Film Co., Ltd. MultiGauge) to measure the sizes of particles and the sintering neck. The spatial resolution was approximately $5 \mathrm{~m}$ for the present experimental setup. The uncertainty for measuring the neck size $x$ was severe in early stage (less than one hour) because the image of the contact point was fuzzy, especially for some particles having rough surface near the contact point. The estimated uncertainties were drawn in $x / r$-time diagrams with error bars. The irregularity of ice particle size was ignored in the 
error estimation due to its small contribution to the uncertainty of $x / r$. One example of measurements of $\mathrm{x}$ is shown in the Electronic Supplementary Information.

Figure 8. Schematic illustration of experimental setup.

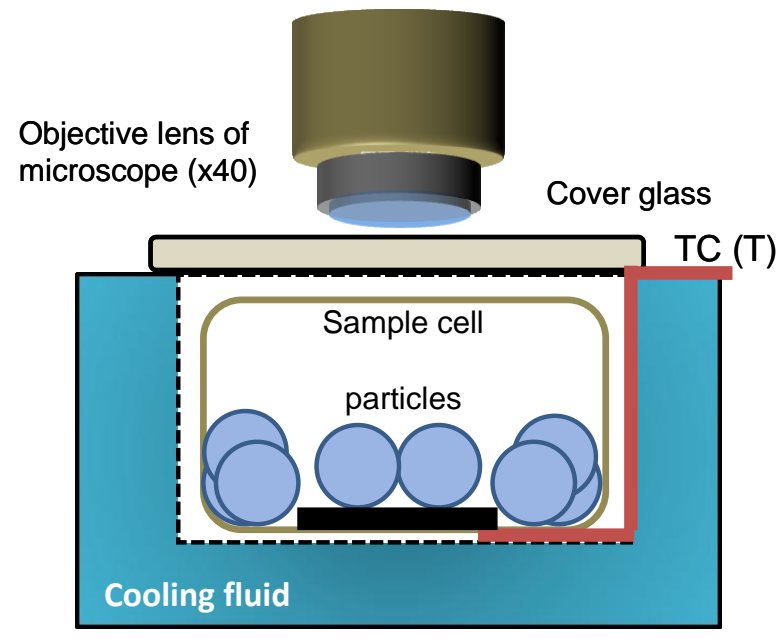

To validate the experimental procedure, the same experiments were carried out on pure ice particles. For experiments under the THF saturation condition, the particle samples were placed on the stage in the sample cell (black rectangle in Figure 8), which was immersed in a small amount of THF solution at the bottom of the cell instead of extra particles. The sample sizes used in the present study are summarized in Table 1.

Table 1. Experimental conditions and rate constants of sintering. $r_{1}$ and $r_{2}$ are the radii of the two particles used in the sintering experiment.

\begin{tabular}{cccc}
\hline & $\boldsymbol{r}_{\mathbf{1}}(\mathbf{m m})$ & $\boldsymbol{r}_{\mathbf{2}}(\mathbf{m m})$ & $\boldsymbol{q}^{\mathbf{1}}$ \\
\hline Ice $(263.1 \mathrm{~K})$ & 1.14 & 1.15 & 0.16 \\
Ice $(257.9 \mathrm{~K})$ & 0.99 & 1.13 & 0.28 \\
THF $(268.5 \mathrm{~K})$ & 1.19 & 1.20 & 0.061 \\
THF $(267.8 \mathrm{~K})$ & 1.09 & 1.10 & 0.015 \\
THF (265.7 K) & 1.19 & 1.25 & 0.037 \\
THF (264.6 K) & 0.92 & 0.97 & 0.049 \\
THF (263.2 K) & 0.79 & 0.82 & 0.055 \\
THF (saturated, 268.2 K) & 1.32 & 1.19 & 0.24 \\
THF (saturated, 262.2 K) & 1.06 & 1.08 & 0.25 \\
THF (saturated, 260.2 K) & 1.07 & 1.18 & 0.31 \\
\hline
\end{tabular}

\section{Conclusions}

We observed the sintering of THF hydrate particles through an optical microscope and confirmed the validity of the experimental procedures using sintering data for ice particles. We then revealed that THF hydrate particles could sinter at a temperature below the melting point of ice. The sintering rate of THF hydrates was lower than that of ice particles at the same temperature and under normal air conditions, but it increased to the same level with ice by the supplementing of THF molecules in vapor. 
This indicates that the sintering rate of THF hydrate particles was controlled by the rate of supplementing guest molecules to the sintering site. This knowledge is useful in considering gas-hydrate processing under storage and transportation conditions.

\section{Acknowledgements}

This study was partially funded by Hokkaido Gas Co., Ltd. We thank Y.H. Mori (Keio University), R. Ohmura (Keio University), H. Hirai (Ehime University), S. Takeya (AIST), B. Kvamme (University of Bergen), C.A. Koh and E.D. Sloan, Jr. (Colorado School of Mines) for their fruitful discussions. We also acknowledge the committee members of the Feasibility Study of Hydrogen Storage Using Gas Hydrates, which was organized by the Geo-space Engineering Center, a division of the Engineering Advancement Association of Japan.

\section{References}

1. Japan Oil, Gas and Metals National Corporation Homepage. http://www.jogmec.go.jp/english/ activities/technology_oil/promoting.html (accessed on 9 December 2010).

2. Fujii, T.; Saeki, T.; Kobayashi, T.; Inamori, T.; Hayashi, M.; Takano, O.; Takayama, T.; Kawasaki, T.; Nagakubo, S.; Nakmizu, M.; Yokoi, K. Resource assessment of methane hydrate by applying a probabilistic approach in the Eastern Nankai Trough, Japan. J. Geogr. 2009, 118, 814-834.

3. Nakata, T.; Hirai, K.; Takaoki, T. Study of natural gas hydrate (NGH) carriers. In Proceedings of the 6th International Conference on Gas Hydrates, Vancouver, Canada, June 2008.

4. Watanabe, S.; Takahashi, S.; Mizubayashi, H.; Mrata, S.; Murakami, H. A demonstration project of NGH land transportation system. In Proceedings of the 6th International Conference on Gas Hydrates, Vancouver, Canada, June 2008.

5. Mao, W.L.; Mao, H.-K., Goncharov, A.F.; Struzhkin, V.V.; Guo, Q.; Hu, J.; Shu, J.; Hemley, R.J.; Somayazulu, M.; Zhao, Y. Hydrogen clusters in clathrate hydrate. Science 2002, 297, 2247-2249.

6. Florusse, L.J.; Peters, C.J.; Schoonman, J.; Hester, K.C.; Koh, C.A.; Dec, S.F.; Marsh, K.N.; Sloan, E.D., Jr. Stable low-pressure hydrogen clusters stored in a binary clathrate hydrate. Science 2004, 306, 469-471.

7. Lee, H.; Lee, J.-W.; Kim, D.Y.; Park, J.; Seo, Y.-T.; Zeng, H.; Moudrakovski, I.L.; Ratcliffe, C.I.; Ripmeester, J.A. Tuning clathrate hydrates for hydrogen storage. Nature 2005, 434, 743-746.

8. Hashimoto, S.; Maruyama, S.; Sugahara, T.; Sato, H.; Ohgaki, K. Thermodynamic and Raman spectroscopic studies on $\mathrm{H}_{2}+$ tetrahydrofuran + water and $\mathrm{H}_{2}+$ tetra- $n$-butyl ammonium bromide + water mixtures containing gas hydrates. Chem. Eng. Sci. 2006, 61, 7884-7888.

9. Strobel, T.A.; Talyor, C.J.; Hester, K.C.; Dec, S.F.; Koh, C.A.; Miller, K.T.; Sloan, E.D., Jr. Molecular Hydrogen Storage in Binary THF- $\mathrm{H}_{2}$ Clathrate Hydrates. J. Phys. Chem. B 2006, 110, 17121-17125.

10. Anderson, R.; Chapoy, A.; Tohidi, B. Phase Relations and Binary Clathrate Hydrate Formation in the System $\mathrm{H}_{2}-\mathrm{THF}-\mathrm{H}_{2} \mathrm{O}$. Langmuir 2007, 23, 3440-3444.

11. Chopoy, A.; Anderson, R.; Tohidi, B. Low-pressure molecular hydrogen storage in semi-clathrate hydrates of quaternary ammonium compounds. J. Am. Chem. Soc. 2007, 129, 746-747. 
12. Shin, K.; Kim, Y.; Strobel, T.A.; Prasad, P.S.R.; Sugahara, T.; Lee, H.; Sloan, E.D. Jr.; Sum, A.K.; Koh, C.A. Tetra- $n$-butylammonium Borohydride Semiclathrate: A Hybrid Material for Hydrogen Storage. J. Phys. Chem. A 2009, 113, 6415-6418.

13. Strobel, T.A.; Hester, K.C.; Sloan, E.D., Jr.; Koh, C.A. A hydrogen clathrate hydrate with cyclohexanone: structure and stability. J. Am. Chem. Soc. 2007, 129, 9544-9545.

14. Prasad, P.S.R.; Sugahara, T.; Lee, H.; Sloan, E.D., Jr.; Sum, A.K.; Koh, C.A. Hydrogen Storage in Double Clathrates with tert-Butylamine. J. Phys. Chem. A 2009, 113, 6540-6543.

15. Hashimoto, S.; Sugahara, T.; Sato, H.; Ohgaki, K. Thermodynamic stability of $\mathrm{H}_{2}+$ tetrahydrofuran mixed gas hydrate in nonstoichiometric aqueous solutions. J. Chem. Eng. Data 2007, 52, 517-520.

16. Struzhkin, V.V.; Militzer, B.; Mao, W.L.; Mao, H.; Hemley, R.J. Hydrogen Storage in Molecular Clathrates. Chem. Rev. 2007, 107, 4133-4151.

17. Mulder, F.M.; Wagemaker, M.; van Eijck, L.; Kearley, G.J. Hydrogen in Porous Tetrahydrofuran Clathrate Hydrate. Chem. Phys. Chem. 2008, 9, 1331-1337.

18. Nakayama, T.; Tomura, S.; Ozaki, M.; Ohmura, R.; Mori, Y.H. Engineering investigation of hydrogen storage in the form of clathrate hydrates: conceptual design of hydrate production plants. Energy Fuel 2010, 24, 2576-2588.

19. Sugahara, T.; Haag, J.C.; Prasad, P.S.R.; Warntjes, A.A.; Sloan, E.D., Jr.; Sum, A.K.; Koh, C.A. Increasing hydrogen storage capacity using tetrahydrofuran. J. Am. Chem. Soc. 2009, 131, 14616-14617.

20. German, R.M. Sintering Theory and Practice; Wiley Interscience: New York, NY, USA, 1996.

21. Blackford, J.R. Sintering and microstructure of ice: a review. J. Phys. D: Appl. Phys. 2007, 40, R355-R385.

22. Talyer, C.J.; Dieker, L.E.; Miller, K.T.; Koh, C.A.; Sloan, E.D., Jr. Micromechanical adhesion force measurements between tetrahydrofuran hydrate particles. J. Colloid Interface Sci. 2007, 306, 255-261.

23. Maeno, N.; Ebinuma, T. Pressure sintering of ice and its implication to the densification of snow at polar glaciers and ice sheets. J. Phys. Chem. 1983, 87, 4103-4110.

24. Kuroiwa, D. A study of ice sintering. Tellus 1961, 13, 252-259.

25. Iwasaki, T.; Takahashi, M.; Katoh, Y.; Horiguchi, K.; Uchida, K. Research on self-preservation of natural gas hydrate pellet (NGHP). Mitsui Zosen Tech. Rev. 2006, 187, 15-21.

26. Hobbs, P.V.; Mason, B.J. The sintering and adhesion of ice. Philos. Mag. 1964, 9, 181-197.

\section{Appendix}

\section{Detailed Process of Measurements from Video Image}

THF hydrate particles were stored in a temperature- controllable cell placed on the stage of the stereoscopic microscope (Nikon FMZ-10) equipped with a CCD camera (Olympus CS230B) and a time-lapse S-VHS video recorder (Victor SR-S990). The spatial resolution was approximately $5 \mu \mathrm{m}$ for the present experimental setup (at $\times 40$ magnification). The lighting of the sample was Nikon cold-fiber optics light system to eliminate the temperature increase during the experiment. 
The analog video-images were transferred to the digital image (I-O data devise, Inc., Magic TV), and analyzed the selected still image $(420 \times 720$ pixels $)$ by using a image analyzing software (Fuji Photo Film Co., Ltd. MultiGauge) to measure the sizes of particles and the sintering neck. Figure S1a shows a still image of the sample. After selecting a close-up image at the sintering place (Figure S1b), we measured the luminosity distribution along the specific line (the red strait line in Figure S1a and S1b). The obtained luminosity distribution for this image is shown in Figure S1c. Then, we obtained the neck size $x$ of the sintering place.

Figure S1. Detailed measurement process from video image. (a) A selected still image from the experiment digital movie transferred from the analog video movie. The square shows the close-up position to observe the neck point. A red line shows the measurement line of luminosity distribution; (b) the close-up image of around the neck point from Figure S1a. The red line is the same as shown in Figure S1a; (c) the obtained luminosity distribution along the red line. The edges of the neck were determined as the position where the luminosity changes sharply.

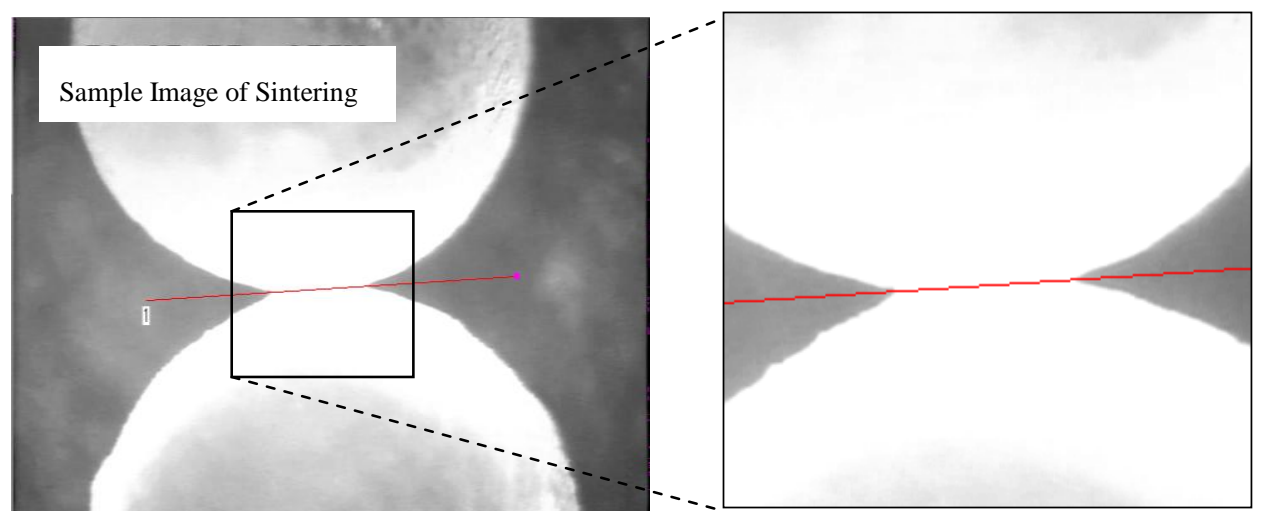

(a)

(b)

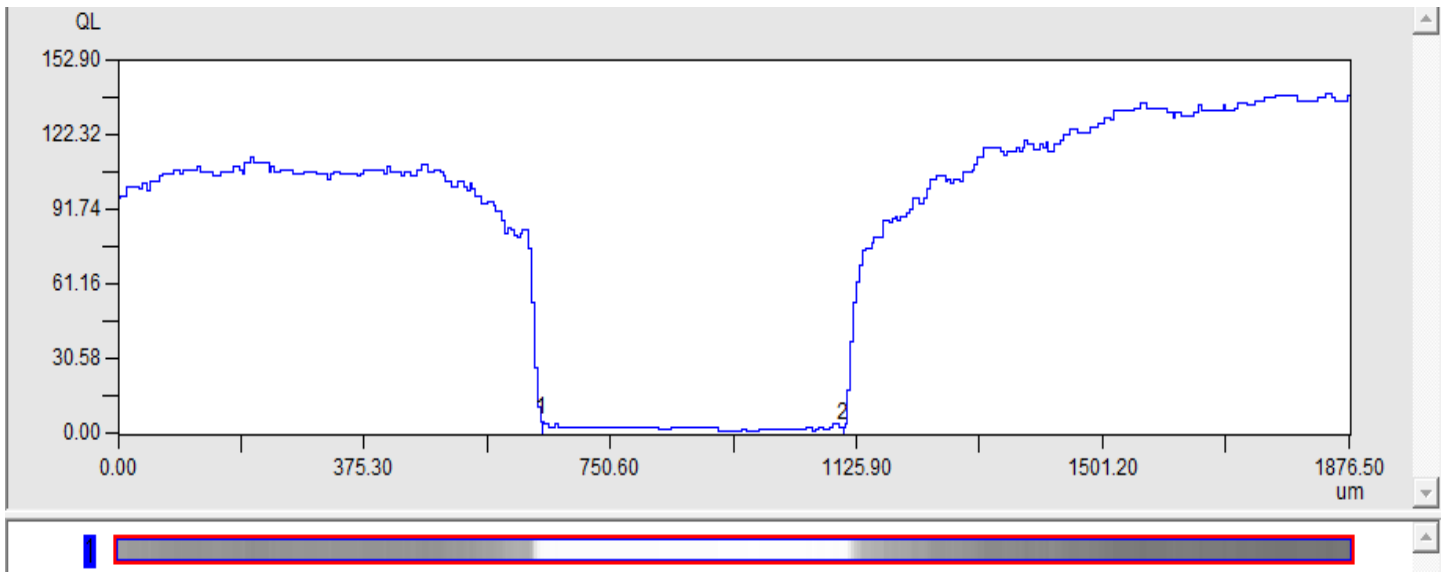

(c)

The uncertainty for measuring the neck size $\boldsymbol{x}$ was severe in early stage (less than one hour) because the image of the contact point was fuzzy, especially for some particles having rough surface near the contact point. The uncertainties in such a case were estimated as the largest difference of $x$ values.

The particle radius $r$ was estimated by a different manner. It was expressed by the radius of the sphere of the equivalent cross section area, and the area of the particle was measured in a still image by 
MultiGauge. At that moment, the magnification of a microscope was reduced to obtain the whole shape of the particle. Although the spatial resolution was also reduced to approximately $9 \mu \mathrm{m}$, the maximum deviation of $r$ from the largest irregularity was almost the same as the spatial resolution. Therefore, the uncertainty of $r$ was enough small to ignore in the estimation of $x / r$.

(C) 2010 by the authors; licensee MDPI, Basel, Switzerland. This article is an open access article distributed under the terms and conditions of the Creative Commons Attribution license (http://creativecommons.org/licenses/by/3.0/). 